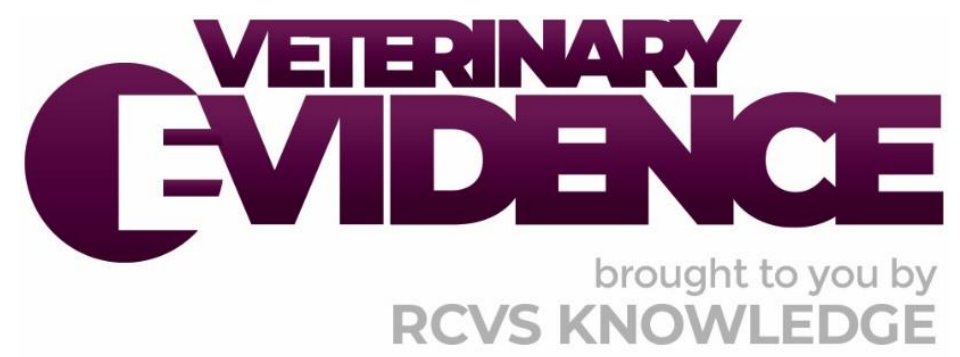

\title{
Are dogs with hip dysplasia in less pain after total hip replacement than femoral head ostectomy?
}

A Knowledge Summary by

Erica R. Rehnblom $D M^{1 *}$

Wanda J. Gordon-Evans DVM PhD DACVS DACVSMR ${ }^{1}$

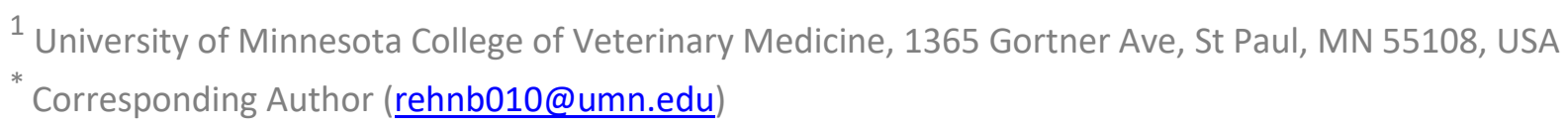

ISSN: 2396-9776

Published: 07 Jan 2022

in: Veterinary Evidence Vol 7, Issue 1

DOI: https://doi.org/10.18849/ve.v7i1.388

Reviewed by: Andy Morris (BSc[Hons] BVSc CertAVP[GSAS]

MRCVS) and Rob Pettitt (BVSc PGCertLTHE

DSAS[Orth] SFHEA FRCVS)

Next Review Date: 08 Oct 2023 


\section{KNOWLEDGE SUMMARY}

\section{PICO question}

In large breed juvenile dogs with hip dysplasia and radiographic bilateral osteoarthritis, is a total hip replacement superior/inferior/or equivalent to bilateral femoral head ostectomy at reducing the severity of long-term hip pain?

\section{Clinical bottom line}

\section{Category of research question}

\section{Treatment}

\section{The number and type of study designs reviewed}

Twelve papers were critically appraised. One paper was a systematic review. Six papers were prospective case series. Five papers were retrospective case series

\section{Strength of evidence}

Weak

\section{Outcomes reported}

Besides one systematic review, there are no other studies available that directly compare pain reduction with total hip replacement and femoral head ostectomy for the treatment of hip dysplasia in large breed juvenile dogs with radiographic evidence of secondary osteoarthritis. In one study, 12/12 (100\%) of owners that responded to an owner outcome questionnaire reported no hip pain with femoral head and neck ostectomy. In this study, owners assessed pain based on activity level of the dog (running, playing, jumping, using stairs normally), gait abnormalities (only when running or after strenuous exercise), and duration of postoperative medications. In eight studies, $91-100 \%$ of cases had no hip pain with total hip replacement reported via clinical examination and/or owner outcome questionnaire

\section{Conclusion}

There is evidence suggesting that both total hip replacement and femoral head ostectomy may be capable of reducing long-term pain as a result of osteoarthritis, secondary to hip dysplasia, however, based on the current literature, it is challenging to say whether total hip replacement is superior to femoral head and neck ostectomy at reducing long-term hip pain. It is important to recognise that other factors considered as outcomes (i.e. range of motion, ground reaction forces, force-plate analysis, etc.) may contribute to differing outcomes overall for total hip replacement vs femoral head ostectomy, but this paper focused specifically on pain. While there is a systematic review that provides evidence supporting that total hip replacement is superior at returning dogs to normal function, evaluating return to normal function was not the focus of this Knowledge Summary

\section{How to apply this evidence in practice}

The application of evidence into practice should take into account multiple factors, not limited to: individual clinical expertise, patient's circumstances and owners' values, country, location or clinic where you work, the individual case in front of you, the availability of therapies and resources.

Knowledge Summaries are a resource to help reinforce or inform decision making. They do not override the responsibility or judgement of the practitioner to do what is best for the animal in their care. 


\section{Clinical Scenario}

You diagnosed a 1 year old female Golden Retriever with bilateral hip dysplasia and persistent hip pain despite appropriate non-surgical management. She shifts her weight forward, bunny hops and has radiographic osteoarthritis. You recommend a total hip replacement or bilateral femoral head ostectomy. The owner wants to know if one option is better at reducing the severity of long-term hip pain.

\section{The evidence}

There is only one prospective case series that directly addresses the present clinical question, however the included population size is very small. Of the literature that solely addresses total hip replacement outcome, five studies are prospective case series and five are retrospective case series. Of the literature that solely addresses femoral head ostectomy outcome, one study is a retrospective case series. There is one systematic review that addresses both total hip replacement and femoral head ostectomy, however return to normal function was their main outcome measurement rather than pain. Overall, because there is only one paper with a small population size directly addressing the present clinical question, the other included literature does not directly compare total hip replacement and femoral head ostectomy for long-term hip pain reduction, and because the current studies all use differing outcome measures and thus a direct comparison cannot be made, it is impossible to draw a meaningful conclusion as to which procedure is more efficacious at reducing longterm hip pain by comparing these studies.

THR - total hip replacement

FHO - femoral head ostectomy

LOAD questionnaire - Liverpool Osteoarthritis in Dogs questionnaire

GRF - ground reaction force

\section{Summary of the evidence}

\begin{tabular}{|c|c|}
\hline \\
\hline \multicolumn{2}{|l|}{$\begin{array}{l}\text { Dueland et al. (1977) } \\
\qquad \text { Population: }\end{array}$} \\
\hline Sample size: & \multirow[b]{2}{*}{$\begin{array}{l}21 \text { dogs. } \\
\text { 17/29 dogs were selected for this study that were }>25 \mathrm{~kg}, \\
\text { had radiographic evidence of dysplasia (except for the four } \\
\text { unaffected dogs), had clinically successful THR and/or FHO, } \\
\text { and had an analysis done after a minimum of } 1 \text { year } \\
\text { postoperatively } \\
\text { - Group } 1 \text { (4/17): normal, unoperated dogs. Group } 2 \text { (4/17): } \\
\text { dogs with unoperated hip vs THR. Group } 3 \text { (5/17): dogs with } \\
\text { one THR and one FHO. Group } 4 \text { (4/17): dogs with bilateral } \\
\text { THR. } \\
\text { Force-plate analysis was done to compare vertical and } \\
\text { horizontal forces. Speed of dog running was obtained by } \\
\text { running them over a known distance and timing this. } \\
\text { - A linear relationship between velocity and force was } \\
\text { established using least squares curve fitting technique. }\end{array}$} \\
\hline & \\
\hline Study design: & Prospective case series. \\
\hline Outcome studied: & $\begin{array}{l}\text { - Objective: force-plate analysis. } \\
\text { - Subjective: clinical evaluation of gait and lameness. }\end{array}$ \\
\hline $\begin{array}{l}\text { Main findings: } \\
\text { (relevant to PICO question): }\end{array}$ & $\begin{array}{l}\text { - When pain is reduced, the landing forces of the limb are } \\
\text { increased. Therefore, if a higher vertical force is apparent on }\end{array}$ \\
\hline
\end{tabular}




\begin{tabular}{|c|c|}
\hline & $\begin{array}{l}\text { one side in an individual, one should assume that this equals } \\
\text { more weight bearing and better performance on that side. } \\
\text { Group 1: normal gait and comparable values between right and } \\
\text { left hips in vertical forces. } \\
\text { - Group 2: in 3/4 dogs, more vertical force was apparent on the } \\
\text { normal, unoperated side. Clinically, all four dogs walked and } \\
\text { ran within normal limits. } \\
\text { - Group 3: all five dogs showed normal gait, } 3 / 5 \text { showed higher } \\
\text { - } \quad \text { Gertical force on the FHO side. } \\
\text { the two hips, } 3 / 4 \text { dogs showed comparable vertical force } \\
\text { between the two hips. } \\
\text { Successful THR may functionally approach a normal hip or } \\
\text { improve a dysplastic hip. } \\
\text { Successful FHO is still a valuable surgical treatment option } \\
\text { which may equal or surpass THR clinically and biomechanically. }\end{array}$ \\
\hline Limitations: & $\begin{array}{l}\text { - Small study population. } \\
\text { - } \quad \text { Case series. } \\
\text { - } \quad \text { Noninicians were unblinded during lameness evaluation. } \\
\text { - }\end{array}$ \\
\hline
\end{tabular}

Olmstead et al. (1983)

\begin{tabular}{|c|c|}
\hline Population: & $\begin{array}{l}\text { Dogs with hip disease (predominantly hip dysplasia) that underwent } \\
\text { THR at The Ohio State University Veterinary Teaching Hospital } \\
\text { (Columbus, Ohio, US) and Berwyn Veterinary Associates Hospital } \\
\text { (Berwyn, Illinois, US). }\end{array}$ \\
\hline Sample size: & 190 dogs. \\
\hline Intervention details: & $\begin{array}{l}\text { - Between August } 1976 \text { and July 1981, } 221 \text { THRs were performed } \\
\text { in } 190 \text { dogs. } \\
\text { - Disabling hip dysplasia was the indication for THR in 182/221 } \\
\text { procedures. } \\
\text { - Physicals, radiographic examination, and owner histories were } \\
\text { used in pre- and yearly postoperative patient evaluation. } \\
\text { - Dogs were evaluated before and yearly thereafter until the dog } \\
\text { died, prosthesis was removed, or the owner could no longer be } \\
\text { contacted. }\end{array}$ \\
\hline Study design: & Prospective case series. \\
\hline Outcome studied: & Subjective: clinical evaluation of lameness, owner questionnaire. \\
\hline $\begin{array}{l}\text { Main findings: } \\
\text { (relevant to PICO question): }\end{array}$ & $\begin{array}{l}\text { - } 149 \text { THRs were still being monitored by January } 1982 \text {, and of } \\
\text { these cases, } 136 / 149 \text { ( } 91.2 \%) \text { had satisfactory results when } \\
\text { function could be evaluated. } \\
\text { - Owners reported satisfactory results as an increase in muscle } \\
\text { mass of the operated leg, and elimination of signs of pain. } \\
\text { - All } 152 \text { dogs with hip dysplasia included in the study were } \\
\text { bilaterally affected, and unilateral replacement was adequate } \\
\text { for } 122 \text { of the dogs. } \\
\text { It is assumed that THR on one side allowed the dog to shift } \\
\text { more of the weight bearing burden in that direction, relieving } \\
\text { pressure on the dysplastic hip. }\end{array}$ \\
\hline
\end{tabular}




\begin{tabular}{|l|l|l|}
\hline & $\begin{array}{l}\text { - Only when a complication was encountered was there a risk } \\
\text { that function would be unsatisfactory. } 46 / 221(20.8 \%) \\
\text { procedures had complications, and } 27 / 46(58.7 \%) \text { ultimately } \\
\text { achieved satisfactory function after revision. }\end{array}$ \\
\hline Limitations: & $\begin{array}{l}\text { - Follow-up could not be completed on the entire population. } \\
\text { - Case series. }\end{array}$ \\
& $\begin{array}{l}\text { Evaluation of function by examiner and owner is very } \\
\text { subjective. }\end{array}$ \\
\hline
\end{tabular}

\begin{tabular}{|c|c|}
\hline \multicolumn{2}{|l|}{ Parker et al. (1984) } \\
\hline Population: & $\begin{array}{l}\text { Dogs with degenerative joint disease secondary to hip dysplasia } \\
\text { underwent THR using a Richards Canine II large size prosthesis. }\end{array}$ \\
\hline Sample size: & 20 dogs. \\
\hline Intervention details: & $\begin{array}{l}\text { - } 17 \text { dogs underwent unilateral THR only, and three dogs } \\
\text { underwent bilateral THR ( } 23 \text { THRs) and were evaluated } \\
\text { before and at } 3 \text { month intervals for } 1 \text { year postoperatively. } \\
\text { - All cases underwent preoperative evaluation which included } \\
\text { a history, complete physical examination, neurological } \\
\text { examination, complete blood count, chemistry panel, and } \\
\text { radiographs. } \\
\text { - All cases had follow-up which consisted of direct observation } \\
\text { or an owner telephone interview. } \\
\text { - The grading system for the affected leg(s) was adapted from } \\
\text { Gendreau \& Cawley (1977) and consisted of excellent (total } \\
\text { weight bearing), good (slight lameness or gait abnormality), } \\
\text { fair (noticeable lameness and may be non-weight bearing } \\
\text { when running), and poor (severe lameness and may be non- } \\
\text { weight bearing at all times. }\end{array}$ \\
\hline Study design: & Prospective case series. \\
\hline Outcome studied: & $\begin{array}{l}\text { Subjective: clinical evaluation of lameness, owner telephone } \\
\text { interview. }\end{array}$ \\
\hline $\begin{array}{l}\text { Main findings: } \\
\text { (relevant to PICO question): }\end{array}$ & $\begin{array}{l}\text { - } 18 / 23(78 \%) \text { of cases were graded as good or excellent. } \\
\text { One patient required revision surgery to achieve a good } \\
\text { outcome. } \\
\text { - } 5 / 23(22 \%) \text { of cases had poor or fair outcomes, and all } \\
\text { developed permanent post-operative complications or had } \\
\text { progression of undiagnosed pre-operative problems } \\
\text { (degenerative myelopathy). }\end{array}$ \\
\hline Limitations: & $\begin{array}{l}\text { - Small study population. } \\
\text { - Case series. } \\
\text { - Clinicians were unblinded during lameness evaluation. } \\
\text { - Subjective owner telephone interviews used to determine } \\
\text { outcome. }\end{array}$ \\
\hline
\end{tabular}




\begin{tabular}{|c|c|}
\hline Population: & Dogs that underwent cemented THR. \\
\hline Sample size: & 84 dogs. \\
\hline Intervention details: & $\begin{array}{l}\text { - Medical records were reviewed for all dogs that underwent } \\
\text { THR from April } 1986 \text { to February } 1992 \text {. Dogs were included } \\
\text { in the study if negative pressure had been applied to the } \\
\text { medullary cavity of the ilium during cementing of the } \\
\text { acetabular component. } \\
\text { - } 96 \text { cases were included. } \\
\text { - } 88 \text { cases were performed secondary to hip dysplasia. } \\
\text { - } 58 \text { of the cases were evaluated by one of the authors or a } \\
\text { referring veterinarian. Mean follow-up time was } 25.1 \\
\text { months (range: } 6-70 \text { months). } \\
\text { - The remaining } 38 \text { cases were evaluated via owner } \\
\text { assessment outcome. Mean follow-up time for these cases } \\
\text { was } 26.7 \text { months (range: } 3-75 \text { months). }\end{array}$ \\
\hline Study design: & Retrospective case series. \\
\hline Outcome studied: & $\begin{array}{l}\text { - Objective: thigh circumference. } \\
\text { - Subjective: clinical evaluation, owner assessment outcome. }\end{array}$ \\
\hline $\begin{array}{l}\text { Main findings: } \\
\text { (relevant to PICO question): }\end{array}$ & $\begin{array}{l}\text { - } 42 / 58 \text { cases clinically evaluated were graded as excellent, } 15 \\
\text { were graded as good. Limb function was graded as poor for } \\
\text { one. } \\
34 \text { of the owner assessment outcome cases were graded as } \\
\text { excellent, one as good, and three as fair. All four dogs with } \\
\text { fair or poor results had complications which necessitated } \\
\text { implant removal. } \\
\text { - Six cases with excellent clinical outcome were selected for } \\
\text { GRF analysis. Two dogs with contralateral degenerative joint } \\
\text { disease had significantly greater vertical force in the THR } \\
\text { limb than for the contralateral limb. The other four dogs had } \\
\text { bilateral THR or unilateral THR with normal contralateral } \\
\text { coxofemoral joint. No significant difference in peak vertical } \\
\text { force in the hindlimbs of these four dogs was detected. } \\
\text { - } 8 / 84 \text { dogs had complications. Four were corrected and hip } \\
\text { function was eventually good or excellent. Implant was } \\
\text { removed in the other four dogs, and final hip function was } \\
\text { fair in three dogs and poor in one. } \\
\text { Cemented THR is an effective treatment for disabling } \\
\text { conditions of the coxofemoral joint in dogs. }\end{array}$ \\
\hline Limitations: & $\begin{array}{l}\text { - Retrospective study design. } \\
\text { - } \text { Case series. } \\
\text { - } \text { Subjective owner assessment outcome. } \\
\text { - Two different THR systems were used. } \\
\text { - Thigh circumference was only measured in good or excellent } \\
\text { results. }\end{array}$ \\
\hline
\end{tabular}




\begin{tabular}{|l|l|}
\hline & $\begin{array}{l}\text { Of the 6/84 dogs that underwent GRF testing, two had } \\
\text { bilateral THR, two had unilateral THR with normal } \\
\text { contralateral coxofemoral joint, and two had unilateral THR } \\
\text { and had contralateral degenerative joint disease. This } \\
\text { variation in disease states can introduce significant bias } \\
\text { when analysing GRF. }\end{array}$ \\
\hline
\end{tabular}

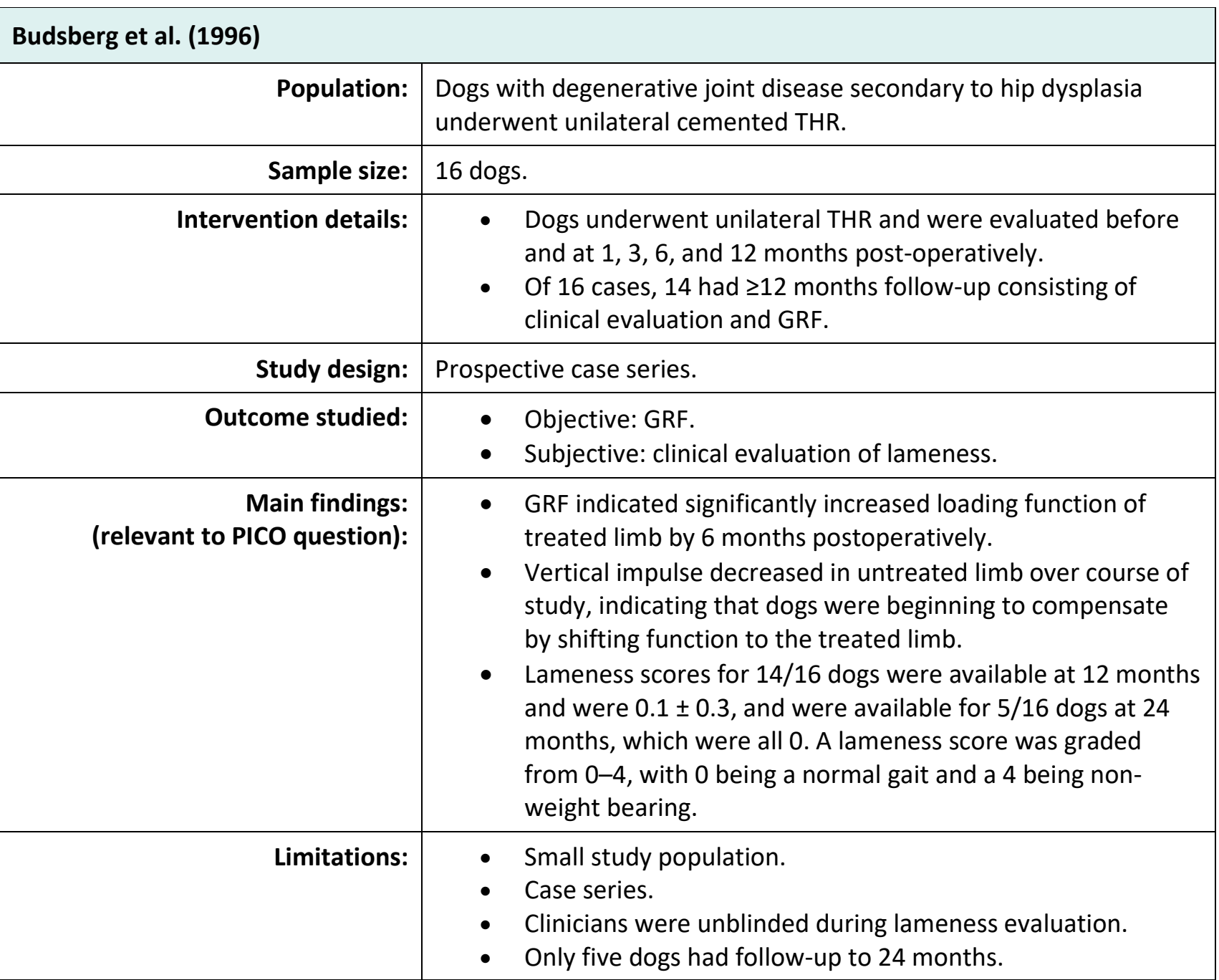

\begin{tabular}{|c|c|}
\hline \\
\hline \multicolumn{2}{|c|}{\begin{tabular}{l|l} 
Population: & $\begin{array}{l}\text { Dogs referred to the Veterinary Specialists of South Florida for } \\
\text { evaluation of hip dysplasia and simultaneous bilateral FHO. }\end{array}$
\end{tabular}} \\
\hline Sample size: & 15 dogs. \\
\hline Intervention details: & $\begin{array}{l}\text { - Medical records were reviewed in all dogs from July } 2000 \text { to } \\
\text { June } 2003 \text { that had radiographic evidence of bilateral } \\
\text { osteoarthritis secondary to hip dysplasia and underwent } \\
\text { simultaneous bilateral FHO. } \\
\text { - } 12 / 15 \text { dogs were evaluated via owner between } 6-48 \text { months } \\
\text { postoperatively via telephone survey. } \\
\text { - Mean age at surgery } 10.3 \text { months (range: } 6-25 \text { months). }\end{array}$ \\
\hline Study design: & Retrospective case series. \\
\hline
\end{tabular}




\begin{tabular}{|c|c|}
\hline Outcome studied: & $\begin{array}{l}\text { - Subjective: owner outcome assessment (time to full } \\
\text { recovery, evidence of pain, and overall satisfaction with the } \\
\text { surgical procedure, concurrent orthopaedic problems). } \\
\text { - Objective: duration and current medications. }\end{array}$ \\
\hline $\begin{array}{l}\text { Main findings: } \\
\text { (relevant to PICO question): }\end{array}$ & $\begin{array}{l}\text { - Five owners graded results as excellent, seven graded the } \\
\text { results as good. } \\
\text { - No dogs had hip pain at time of follow-up. } \\
\text { - Seven dogs had slightly abnormal gait only when running or } \\
\text { after strenuous exercise. }\end{array}$ \\
\hline Limitations: & $\begin{array}{l}\text { - } \quad \text { Retrospective study design. } \\
\text { - } \quad \text { Case series. } \\
\text { - } \quad \text { Subjective owner outcome assessment. } \\
\text { - } \quad \text { All dogs were offered follow-up examination and } \\
\text { - } \quad \text { radiographs. These were only performed in four dogs. } \\
\text { - } 12 / 15(80 \%) \text { of owners responded in a small study size. }\end{array}$ \\
\hline
\end{tabular}

\begin{tabular}{|c|c|}
\hline \\
\hline \multicolumn{2}{|l|}{$\begin{array}{l}\text { Guerrero et al. (2009) } \\
\text { Population: }\end{array}$} \\
\hline Sample size: & \multirow[b]{2}{*}{$\begin{array}{l}60 \text { dogs. } \\
\text { - 59/65 THRs were completed between April } 2001 \text { and } \\
\text { September } 2003 \text { on dogs with hip dysplasia and secondary } \\
\text { coxoarthrosis. } \\
\text { - Information obtained included breed, sex, body weight, date } \\
\text { of surgery, indication for THR, operated side, surgical time, } \\
\text { angles lateral opening (ALO), inclination of cup (Al), longest } \\
\text { clinical and radiographic follow-up, intra- and postoperative } \\
\text { complications, management of complications, and outcome. } \\
\text { - One surgeon performed all THRs. } \\
\text { - All dogs had postoperative evaluation which included clinical } \\
\text { evaluation (pain on manipulation of hip joint, range of } \\
\text { motion, muscle mass compared with contralateral leg, } \\
\text { lameness) } \geq 6 \text { months. } \\
\text { Clinical evaluation was scored based on a previously } \\
\text { reported scale, of excellent, good, fair, poor, or failed } \\
\text { function. }\end{array}$} \\
\hline Intervention details: & \\
\hline Study design: & Prospective case series. \\
\hline Outcome studied: & Subjective: clinical evaluation of lameness. \\
\hline $\begin{array}{l}\text { Main findings: } \\
\text { (relevant to PICO question): }\end{array}$ & $\begin{array}{l}\text { - 60/65 THRs were considered to have excellent clinical } \\
\text { outcome, three had good outcome, and two as failed } \\
\text { outcome. } \\
\text { - High complication rates, } 11 / 65 \text { (17\%), consisted } \\
\text { predominantly of THR luxations, } 7 / 65 \text { (11\%). } \\
\text { - After revision, a successful outcome was achieved in } 63 / 65 \\
\text { (97\%) of THRs. }\end{array}$ \\
\hline Limitations: & \\
\hline
\end{tabular}




\begin{tabular}{|l|l|}
\hline & $\begin{array}{l}\text { - Case series. } \\
\text { - Clinicians were unblinded during lameness evaluation. } \\
\text { - Previously reported clinical evaluation scale was not } \\
\text { disclosed. }\end{array}$ \\
\hline
\end{tabular}

\begin{tabular}{|c|c|}
\hline \multicolumn{2}{|l|}{ Gemmill et al. (2011) } \\
\hline Population: & $\begin{array}{l}\text { Dogs undergoing hybrid THR using a cementless acetabular } \\
\text { component and a cemented femoral component. }\end{array}$ \\
\hline Sample size: & 71 dogs. \\
\hline Intervention details: & $\begin{array}{l}\text { - Dogs were selected for the study from December } 2005 \text { to } \\
\text { July } 2009 \text { if they had debilitating hip disease unresponsive to } \\
\text { conservative management and absence of concurrent } \\
\text { medical, orthopaedic, or neurologic disease that would } \\
\text { preclude surgery. } \\
\text { - Follow-up } \geq 6 \text { months. } \\
\text { - Of } 78 \text { procedures, hip dysplasia was indicated in } 68 \text {. } \\
\text { - Dogs were evaluated at } 4 \text { and } 12 \text { weeks, and } 6-27 \text { months } \\
\text { by veterinarians. } \\
\text { - Owner outcome assessment completed } 6-40 \text { months } \\
\text { postoperatively. }\end{array}$ \\
\hline Study design: & Prospective case series. \\
\hline Outcome studied: & $\begin{array}{l}\text { Subjective: owner outcome assessment, pain upon manipulation by } \\
\text { veterinarian. }\end{array}$ \\
\hline $\begin{array}{l}\text { Main findings: } \\
\text { (relevant to PICO question): }\end{array}$ & $\begin{array}{l}\text { - Pain, lameness, and disability were reported to be mild in } \\
3 / 78(3.8 \%) \text { cases and absent in } 75 / 78(96.2 \%) \text { cases. } \\
\text { - } 73 / 78(94 \%) \text { of cases were reported to have normal quality } \\
\text { of life at owner assessment outcome. } \\
\text { - Of four cases that had postoperative complications, three } \\
\text { were successfully revised. }\end{array}$ \\
\hline Limitations: & $\begin{array}{l}\text { - Case series. } \\
\text { - Veterinarian evaluation was done by the vet who performed } \\
\text { the procedure, thus bias may be introduced by multiple } \\
\text { examiners. } \\
\text { - Subjective owner outcome assessment. }\end{array}$ \\
\hline
\end{tabular}

Bergh et al. (2014)

\begin{tabular}{|r|l|}
\hline Population: & $\begin{array}{l}\text { Dogs with naturally occurring canine hip dysplasia that were treated } \\
\text { with various surgical procedures. }\end{array}$ \\
\hline Sample size: & 848 dogs. \\
\hline Intervention details: & $\begin{array}{l}\text { Of } 477 \text { manuscripts, } 17 \text { met inclusion criteria. These were } \\
\text { grouped based on surgical procedure (THR, triple pelvic } \\
\text { osteotomy, juvenile pubic symphysiodesis, Chiari } \\
\text { osteotomy/intertrochanteric osteotomy, and FHO) and level } \\
\text { of evidence (I-V) relative to the study question. }\end{array}$ \\
\hline
\end{tabular}




\begin{tabular}{|c|c|}
\hline & $\begin{array}{l}\text { - There were seven total hip replacement studies and one } \\
\text { femoral head ostectomy study. Unilateral surgeries with }>6 \\
\text { months follow-up were included. } \\
\text { - The outcome measurements included were orthopaedic } \\
\text { exam, owner interview, visual gait observation, and force } \\
\text { plate gait analysis depending on the study. }\end{array}$ \\
\hline Study design: & Systematic literature review. \\
\hline Outcome studied: & $\begin{array}{l}\text { - Subjective: orthopaedic examination, owner } \\
\text { interview/questionnaire, and/or visual gait observation. } \\
\text { - Objective: force plate gait analysis. }\end{array}$ \\
\hline $\begin{array}{l}\text { Main findings: } \\
\text { (relevant to PICO question): }\end{array}$ & $\begin{array}{l}\text { - THR consistently returned dogs to normal function. } \\
\text { - FHO did not consistently return dogs to normal function. }\end{array}$ \\
\hline Limitations: & $\begin{array}{l}\text { - The review was in depth but the studies measured mostly } \\
\text { subjective outcomes. } \\
\text { - All THR and FHO studies had low levels of evidence. } \\
\text { - Comparison between procedures was difficult due to varying } \\
\text { outcome measurements. } \\
\text { - The review assesses functional outcome instead of strictly } \\
\text { pain outcome. } \\
\text { - Three total hip replacement studies had level III evidence, } \\
\text { four had level IV evidence. } \\
\text { - The only FHO study had level IV evidence. }\end{array}$ \\
\hline
\end{tabular}

\section{Fitzpatrick et al. (2014)}

\begin{tabular}{|c|c|}
\hline Population: & $\begin{array}{l}\text { Skeletally immature dogs with hip dysplasia undergoing THR using } \\
\text { BioMedtrix BFXTM biologic fixation implants after unsatisfactory } \\
\text { outcome with medical management. }\end{array}$ \\
\hline Sample size: & 20 dogs. \\
\hline Intervention details: & $\begin{array}{l}\text { Medical records were reviewed from November } 2007 \text { to } \\
\text { June } 2010 \text { and dogs that had unsatisfactory outcome with } \\
\text { medical management due to coxofemoral pain resulting } \\
\text { from canine hip dysplasia that underwent unilateral } \\
\text { cementless THR using BFXTM implants were selected for the } \\
\text { study. } \\
\text { - All dogs at time of surgery were } 6-10 \text { months of age. } \\
\text { - All dogs were available for follow-up immediately and } 6 \\
\text { weeks postoperatively, } 19 \text { were available for long-term } \\
\text { follow-up. }\end{array}$ \\
\hline Study design: & Retrospective case series. \\
\hline Outcome studied: & $\begin{array}{l}\text { Subjective: owner outcome assessment via questionnaire at final } \\
\text { follow-up (function, pain, and analgesia requirements), veterinary } \\
\text { examination score (maximum possible score of } 11 \text {, including } \\
\text { lameness, musculature, pain, and range of motion) immediately, at } 6 \\
\text { weeks, and at long-term follow-up (mean of } 29.8 \text { months, range: } 12- \\
48 \text { months). }\end{array}$ \\
\hline $\begin{array}{l}\text { Main findings: } \\
\text { (relevant to PICO question): }\end{array}$ & $\begin{array}{l}\text { - Mean veterinary examination score } 0.8 \text { (range: } 0-4 \text { ) at long- } \\
\text { term follow-up. }\end{array}$ \\
\hline
\end{tabular}




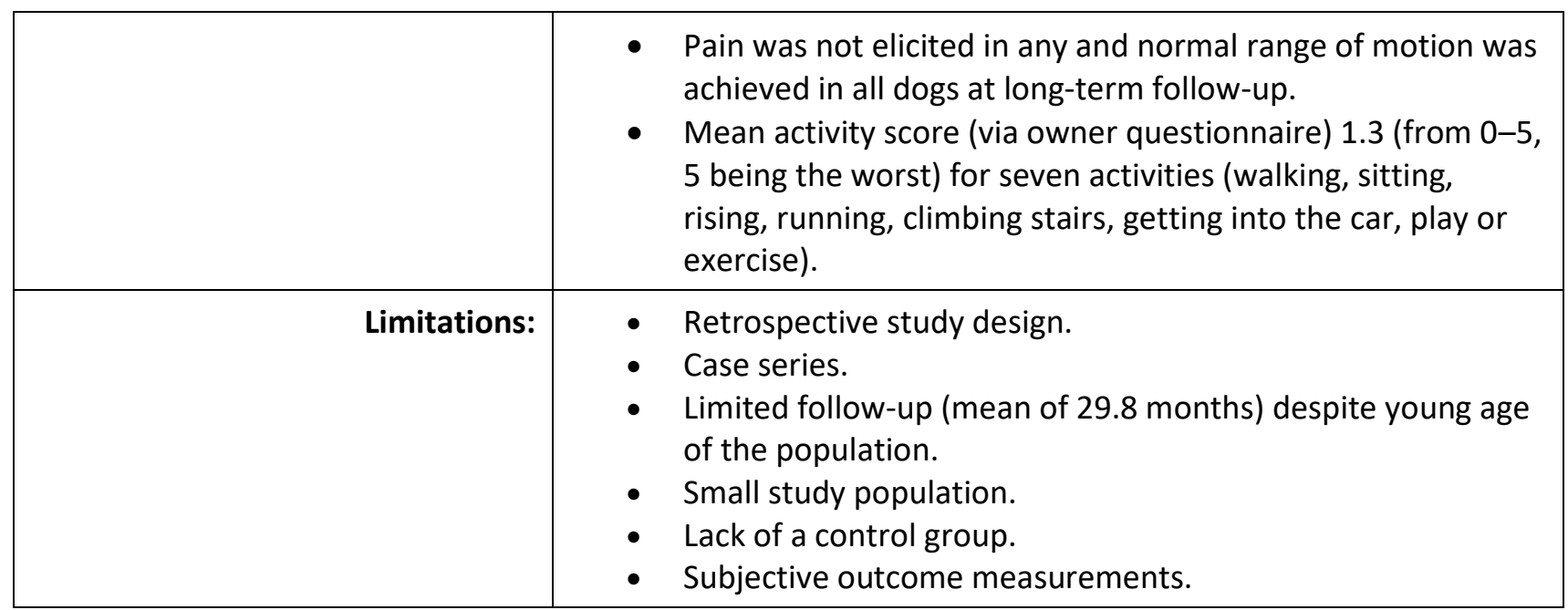

\begin{tabular}{|c|c|}
\hline \multicolumn{2}{|l|}{ Vezzoni et al. (2015) } \\
\hline Population: & $\begin{array}{l}\text { Dogs that underwent Zürich cementless THR at the Clinica } \\
\text { Veterinaria Vezzoni S.R.L, Cremona, Italy. }\end{array}$ \\
\hline Sample size: & 321 dogs. \\
\hline Intervention details: & $\begin{array}{l}\text { - Records were reviewed from January } 2002 \text { to December } \\
2007 \text { and of } 348 \text { dogs and } 479 \text { Z-THAs, } 321 \text { dogs and } 439 \\
\text { cases fit the inclusion criteria. This included } 191 \text { juvenile } \\
\text { cases, } 248 \text { adult cases, and } 118 \text { bilateral cases treated for } \\
\text { hip dysplasia. } \\
\text { - Dogs with a follow-up } \geq 2 \text { years were included. } \\
\text { - Juvenile group } \leq 11 \text { months old. } \\
\text { - Adult group } \geq 11 \text { months old. } \\
\text { - Follow-up was done at } 2,6 \text {, and } 12 \text { months postoperatively, } \\
\text { and yearly thereafter with physical examination. } \\
\text { - Any outcome other than a normally functioning hip was } \\
\text { classified as a complication. }\end{array}$ \\
\hline Study design: & Retrospective case series. \\
\hline Outcome studied: & $\begin{array}{l}\text { - Objective: weight change after surgery } \\
\text { - Subjective: clinical examination including body condition } \\
\text { score, muscle mass, range of motion, presence of any pain } \\
\text { or discomfort during manipulation, and video recording of } \\
\text { gait. }\end{array}$ \\
\hline $\begin{array}{l}\text { Main findings: } \\
\text { (relevant to PICO question): }\end{array}$ & $\begin{array}{l}\text { - Adult group: } 33 / 238 \text { (14\%) complications, } 31 \text { successfully } \\
\text { revised, two explanted. } 10 \text { cases were not accounted for. } \\
\text { - Juvenile group: } 39 / 191 \text { (20\%) complications, } 37 \text { successfully } \\
\text { revised, two explanted. } \\
\text { - Difference in complication rate not statistically significant. }\end{array}$ \\
\hline Limitations: & $\begin{array}{l}\text { - Retrospective study design. } \\
\text { - } \text { Case series. } \\
\text { - Twbjective outcome measurements. } \\
\text { implants for biomechanical studies. }\end{array}$ \\
\hline
\end{tabular}




\begin{tabular}{|c|c|}
\hline \multicolumn{2}{|l|}{ Bayer et al. (2019) } \\
\hline Population: & $\begin{array}{l}\text { Dogs with hip dysplasia that underwent THR using a combined } \\
\text { implant system consisting of INNOPLANT Screw Cup, KYON taper } \\
\text { head, and Zürich cementless (Z-THR) stem. }\end{array}$ \\
\hline Sample size: & 12 dogs. \\
\hline Intervention details: & $\begin{array}{l}\text { - Records were reviewed from March } 2010 \text { to March } 2015 \\
\text { and } 16 \text { hybrid THR procedures were performed in } 12 \text { dogs. } \\
\text { - Follow-up examination was performed at } 2,6 \text {, and } 12 \\
\text { months postoperatively. } \\
\text { - } \quad \text { Follow-up } \geq 2 \text { years. } \\
\text { - Mean age at surgery } 31 \text { months (range: } 12-98 \text { months). }\end{array}$ \\
\hline Study design: & Retrospective case series. \\
\hline Outcome studied: & $\begin{array}{l}\text { Objective: owner-administered outcome LOAD assessment. } \\
\text { Osteoarthritis was defined as mild for LOAD scores 0-10, } \\
\text { moderate for 11-20, severe for 21-30, and extreme from } \\
31-52 \text {. } \\
\text { - Subjective: clinical outcome examination including pain or } \\
\text { discomfort upon manipulation of hip joint, range of motion, } \\
\text { lameness grade (0-4), and thigh circumference compared to } \\
\text { contralateral limb. }\end{array}$ \\
\hline $\begin{array}{l}\text { Main findings: } \\
\text { (relevant to PICO question): }\end{array}$ & $\begin{array}{l}\text { - } 15 \text { cases had full function and one had acceptable function } \\
\text { at final follow-up. } \\
\text { - In dogs with full function, all had thigh circumference that } \\
\text { was bilaterally symmetrical. } \\
\text { - Median LOAD score for procedures with full functional } \\
\text { outcome was } 5 \text { (range: } 3-11 \text { ). } \\
\text { - Three major complications occurred in three cases, all had } \\
\text { complete revision and resolution. }\end{array}$ \\
\hline Limitations: & $\begin{array}{l}\text { - Retrospective study design. } \\
\text { - } \text { Case series. } \\
\text { - } \text { Small study population. } \\
\text { limited experience (the surgeon had performed } 28 \text { THA } \\
\text { procedures previously, compared to the proposed initial } \\
\text { learning curve of a minimum of } 44 \text { THA procedures), which } \\
\text { may introduce surgeon-related complications. } \\
\text { - Subjective outcome measurements. }\end{array}$ \\
\hline
\end{tabular}

\section{Appraisal, application and reflection}

After a thorough search of the literature, only one paper was found that specifically addresses the PICO question, and twelve papers were found that partially address it. Included in this Knowledge Summary is one systematic review, six prospective case series, and five retrospective case series. Unfortunately, other than the systematic analysis and one prospective case series with force-plate analysis and very small population size, none of the other studies directly compare THR and FHO, and differing outcome measures used throughout the studies make it difficult to draw a meaningful conclusion in regards to the clinical question.

The strongest evidence available comes from the systematic review (Bergh et al., 2014). Included in the systematic review are seven manuscripts regarding THR and one manuscript regarding FHO. In regards to the 
clinical question, THR consistently returned dogs to normal limb function while FHO did not consistently return dogs to normal limb function. However, it is important to note that while the systematic review represents the highest level of evidence in this study, it is not without limitations. The systematic review focused on $\operatorname{limb}$ functionality, which only indirectly relates to the present clinical question in regards to reducing the severity of long-term pain. It is also important to point out that the manuscripts evaluated in the systematic review used differing outcome measurements and had low levels of evidence, which means that their conclusion must be interpreted with caution.

One prospective case series (Gemmill et al., 2011) analysed 71 dogs for a total of 78 hybrid THR replacement procedures with a cementless acetabular component and a cemented femoral component with follow-up of $\geq$ 6 months. At time of owner outcome assessment, 75/78 (96\%) of dogs were reported to have no hip pain. This subjective outcome measure of owner outcome assessment and lack of a control group weakens the evidence of this study. A second prospective case series (Budsberg et al., 1996) analysed 16 dogs for a total of 16 unilateral cemented total hip replacement procedures, 14 of which had follow-up $\geq 12$ months. All dogs in this study had significantly increased GRF for loading function of the treated limb by 6 months postoperatively. Five dogs had follow-up at 24 months, and at this time all five dogs had lameness scores of 0 . This study represents one of the few studies with objective outcome measurements in the form of GRF, and thus strengthens the evidence for THR reducing the severity of long-term hip pain as a result of hip dysplasia. A third prospective case series (Olmstead et al., 1983) analysed 221 THRs in 190 dogs performed predominantly as a result of hip dysplasia using physicals, radiographic examination, and owner histories in pre- and yearly post-operative patient evaluation. 136/149 (91.2\%) of THRs had satisfactory function at time of follow-up suggesting that THR may reduce the severity of long-term hip pain as a result of hip dysplasia, however the subjective outcome of clinical evaluation and owner assessment weaken the evidence of this study. A fourth prospective case series (Parker et al., 1984) analysed 20 dogs with 23 THRs using a Richards Canine II large size prosthesis. Outcome evaluation consisted of direct observation and owner telephone outcome assessment. Through direct observation, THR outcome was graded using a grading system for the affected leg(s) that was adapted from Gendreau \& Cawley (1977) and consisted of excellent (total weight bearing), good (slight lameness or gait abnormality), fair (noticeable lameness and may be non-weight bearing when running), and poor (severe lameness and may be non-weight bearing at all times. $18 / 23(78 \%)$ of THRs had excellent or good outcome. Subjective observation and owner telephone assessment weaken the evidence that THRs provide excellent to good outcome for decreasing pain in dogs with hip dysplasia, however using a standardised grading system minimises subjective grading bias. A fifth prospective case series (Guerrero et al., 2009) anaylsed 60 dogs that underwent 65 THRs using 2 nd generation Zurich cementless THR. All dogs had postoperative evaluation which included clinical evaluation (pain on manipulation of hip joint, range of motion, muscle mass compared with contralateral leg, lameness) $\geq 6$ months, and clinical evaluation was scored based on a previously reported scale, of excellent, good, fair, poor, or failed function. 60/65 (92.3\%) of THRs were considered to have excellent clinical outcome. Using a standardised scale for clinical evaluation minimises subjective clinical evaluation, however the scale used was not reported, weakening the evidence supporting that THRs decrease long-term pain associated with THR. Finally, a sixth prospective case series (Dueland et al., 1977) analysed 21 dogs, 17 of which had degenerative joint disease secondary to hip dysplasia that underwent either THR and/or FHO, and four unaffected dogs as controls. Dogs were divided into four groups, group 1 was normal, unoperated dogs, group 2 was dogs with one unoperated hip vs one THR, group 3 was dogs with one THR and one FHO, and group 4 was dogs with bilateral THRs. Force-plate analysis was done to compare vertical and horizontal forces, and clinical evaluation of gait and lameness was also reported. Based on forceplate analysis, THR may functionally approach a normal hip or improve a dysplastic hip, and successful FHO may equal or surpass THR clinically and biomechanically. This study provides strong objective analysis by using force-plate technology suggesting that THR and FHO may improve gait, lameness, and force of operated limb. However, the sample size was incredibly low for each group (4-5 dogs) so a larger study should be performed before these results can be more widely accepted.

There was one retrospective case series (Bayer et al., 2019) that analysed 12 dogs for a total of 16 THR procedures using a combined implant system consisting of INNOPLANT Screw Cup, KYON taper head, and Zürich cementless (Z-THR) stem. These cases were evaluated with clinical examination and LOAD 
questionnaires performed by the owners. $15 / 16$ cases had full function while $1 / 16$ cases had acceptable function. Median LOAD score was 5 (range: 3-11). The LOAD score is a standardised tool for collecting data from owners, and in this case supported the subjective clinical examination findings and outcome determined at the final follow-up visit that THR is efficacious at reducing the severity of long-term hip pain associated with hip dysplasia. Although the retrospective and subjective outcome measures weaken the evidence of this study, this is the only retrospective case study out of five that used a standardised owner outcome questionnaire.

Of the three remaining retrospective case series regarding THR, one study (Vezzoni et al., 2015) analysed 439 Zürich cementless THR procedures and all dogs except for four had normal clinical outcome via clinical examination at their 12 month follow-up, and the remaining two studies (Massat et al., 1994; and Fitzpatrick et al., 2014) used both clinical examination and owner assessment outcome as outcome measurements, and found $84 / 88$ cases had good or excellent outcomes, and 20/20 cases had no pain upon clinical examination respectively.

There were only three studies for FHO outcome that related to the present clinical question. The first (Rawson et al., 2005) as discussed above, found that all dogs had no hip pain at time of follow-up according to owner questionnaire. The second study (Ganesh et al., 2017) was unavailable and thus is not included in the answering of the present clinical question. The third study (Dueland et al., 1977) used force-plate analysis to determine that successful FHO may improve gait, lameness, and force of operated limb, which may be interpreted as decreased pain, however the study population was very small. Unfortunately, FHO studies are lacking, which contributes to the difficulty in answering the present clinical question. Two studies (Off \& Matis et al., 2010; and Duff et al., 1977) discussing FHO outcome were not included in this Knowledge Summary due to Legg-Calvé Perthes disease being the predominant indication for surgery rather than hip dysplasia, presenting the possibility that femoral head necrosis may affect FHO outcome differently than FHO outcome due to hip dysplasia.

From the available data, it is possible that both THR and FHO may reduce the severity of long-term pain as a result of osteoarthritis secondary to hip dysplasia given the owner assessment outcomes and clinical evaluation outcomes in the aforementioned studies. However, given the differing outcome measurements between studies such as subjective non-standardised owner assessment outcomes, subjective clinical evaluation, GRF, and force-plate analysis it is impossible to compare one study to another. It is also important to mention varying postoperative pain management and physical rehabilitation not only between different studies but between individual patients in a given study as an additional variable influencing outcome and ultimately long-term pain associated with FHO and THR. It is also important to note that there are several more studies analysing THR than FHO, which provides an unequal amount of evidence towards the success of THR at reducing long-term pain associated with hip dysplasia. From the evidence, it appears that THR provides a successful outcome in many cases, however it is not evident whether THR provides a superior/inferior/or equivalent outcome compared to FHO given the lack of studies analysing FHO. In order to definitively answer the present clinical question, a prospective, randomised clinical trial, with pre-determined standardised outcome measurements, comparing THR treated dogs to FHO treated dogs with naturally occurring hip dysplasia would be necessary.

Two papers (Vezzoni et al., 2015; and Bayer et al., 2019) were not indexed by either database using the below search terms, however both papers were deemed applicable based on reference checking. This represents an inherent bias of the chosen databases, and represents a limitation of this Knowledge Summary. 


\section{Methodology Section}

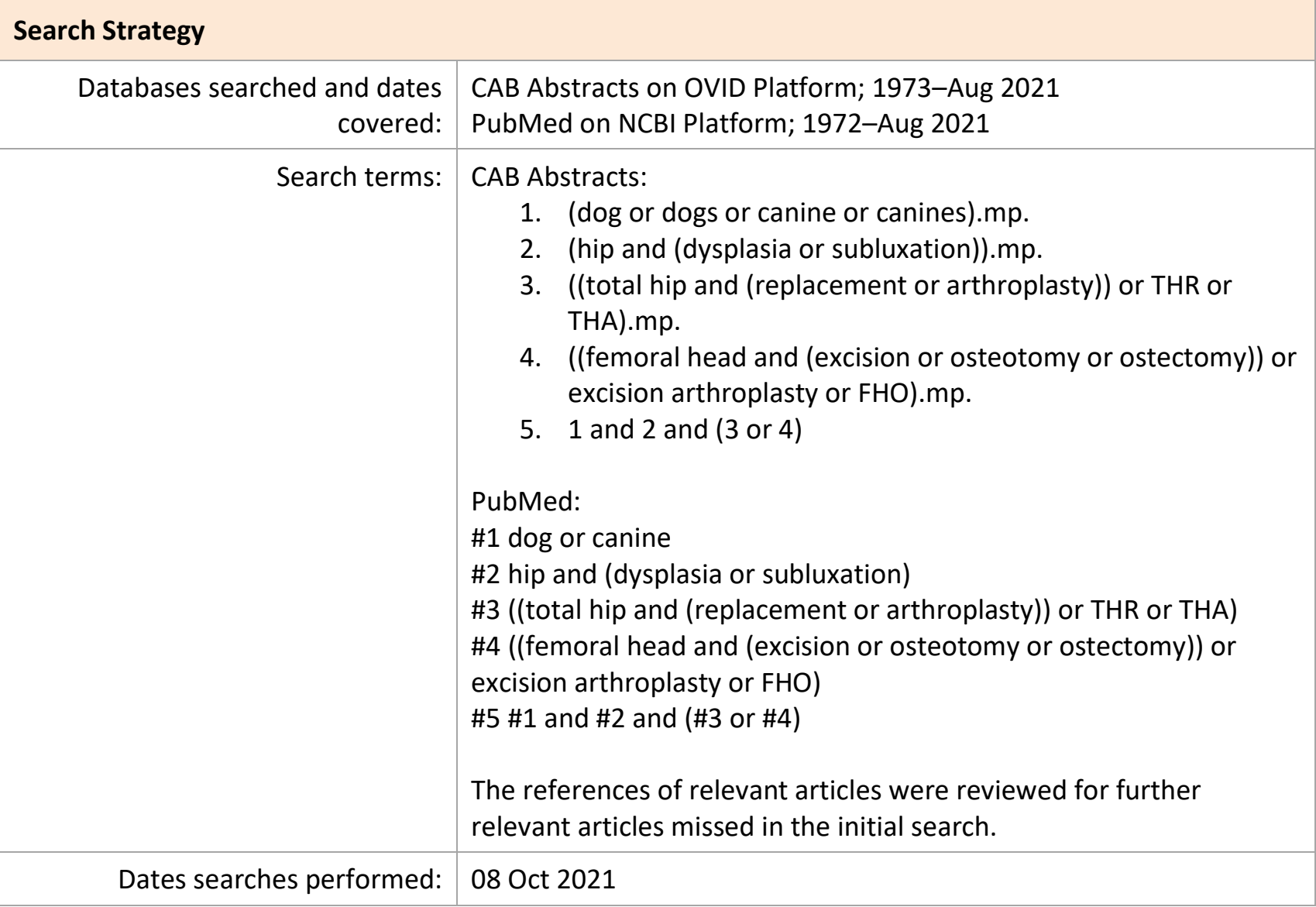

\section{Exclusion / Inclusion Criteria}

Exclusion: Book chapters, conference proceedings, articles not available in English, clinical reviews, case studies, studies with fewer than 10 dogs at time of follow-up, studies using prototypic hip replacement models, biceps femoris muscle sling technique for FHO.

Inclusion: Articles written in English relevant to the PICO question, studies with $\geq 6$ months follow-up for all included cases, studies evaluating treatment primarily for hip dysplasia (hip dysplasia as the majority of cases included in the study), commercially available total hip replacement implants. 


\begin{tabular}{|l|c|c|c|c|c|}
\hline \multicolumn{1}{|c|}{ Search Outcome } & \multicolumn{2}{|l|}{} \\
\hline \multicolumn{1}{|c|}{ Database } & $\begin{array}{c}\text { Number } \\
\text { of results }\end{array}$ & $\begin{array}{c}\text { Excluded - } \\
\text { Reviews }\end{array}$ & $\begin{array}{c}\text { Excluded - Not } \\
\text { available in English }\end{array}$ & $\begin{array}{r}\text { Excluded - Not } \\
\text { relevant to PICO } \\
\text { question }\end{array}$ & $\begin{array}{c}\text { Total } \\
\text { relevant } \\
\text { papers }\end{array}$ \\
\hline CAB Abstracts & 139 & 26 & 30 & 76 & 6 \\
\hline $\begin{array}{l}\text { PubMed } \\
\text { Reference }\end{array}$ & 105 & 14 & 3 & 80 & 2 \\
\hline Checking & 6 & 0 & 0 & 4 & 12 \\
\hline
\end{tabular}

\section{CONFLICT OF INTEREST}

The authors declare no conflict of interest.

\section{REFERENCES}

1. Bayer, K., Matiasovic, M., Steger, H. \& Böttcher, P. (2019). Complications and Long-Term Outcome in 16 Canine Cementless Hybrid Hip Arthroplasties. Veterinary and Comparative Orthopaedics and Traumatology.32(1), 73-78. DOI: https://doi.org/1055/s-0038-1676072

2. Bergh, M. S. \& Budsberg, S. C. (2014). A Systematic Review of the Literature Describing the Efficacy of Surgical Treatments for Canine Hip Dysplasia (1948-2012). Veterinary Surgery.43(5), 501-506.

DOI: https://doi.org/10.1111/i.1532-950X.2014.12208.x

3. Budsberg, S. C., Chambers, J. N., Lue, S. L. van, Foutz, T. L. \& Reece, L. (1996). Prospective evaluation of ground reaction forces in dogs undergoing unilateral total hip replacement. American Journal of Veterinary Research. 57(12), 1781-1785.

4. Dueland, R., Bartel, D. L. \& Antonson, E. (1977). Force-plant technique for canine gait analysis of total hip and excision arthroplasty [proceedings]. Journal of the American Animal Hospital Association. 13(5), 547-552.

5. Duff, R. \& Campbell, J. R. (1977). Long term results of excision arthroplasty of the canine hip. The Veterinary Record. 101(10), 181-184.

6. Fitzpatrick, N., Law, A. Y., Bielecki, M. \& Girling, S. (2014). Cementless Total Hip Replacement in 20

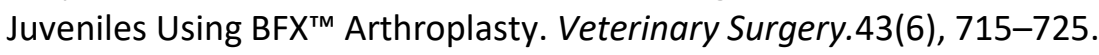

DOI: https://doi.org/10.1111/i.1532-950X.2014.12214.x

7. Ganesh, T. N., Siobhan Bridglalsingh \& Legall, C. (2017). Femoral head and neck excision arthroplasty in 38 dogs: a two-year study with special reference to surgical indication and outcome. Indian Journal of Veterinary Surgery, 38(2), 77-80. [online] Available from: http://ovidsp.ovid.com/ovidweb.cgi?T=JS\&PAGE=reference\&D=caba6\&NEWS=N\&AN=20193238 298 [Accessed 08 Aug 2021] 
8. Gemmill, T. J., Pink, J., Renwick, A., Oxley, B., Downes, C., Roch, S. \& McKee, W. M. (2011). Hybrid Cemented/Cementless Total Hip Replacement in Dogs: Seventy-Eight Consecutive Joint Replacements. Veterinary Surgery.40(5), 621-630. DOI: https://doi.org/10.1111/j.1532950X.2011.00827.x

9. Guerrero, T. G. \& Montavon, P. M. (2009). Zurich Cementless Total Hip Replacement: Retrospective Evaluation of 2 nd Generation Implants in 60 Dogs. Veterinary Surgery.38(1), 70-80. DOI: https://doi.org/10.1111/i.1532-950X.2008.00466.x

10. Massat, B. J. \& Vasseur, P. B. (1994). Clinical and radiographic results of total hip arthroplasty in dogs: 96 cases (1986-1992). Journal of the American Veterinary Medical Association. 205(3), 448-454.

11. Off, W. \& Matis, U. (2010). Excision arthroplasty of the hip joint in dogs and cats. Clinical, radiographic, and gait analysis findings from the Department of Surgery, Veterinary Faculty of the LudwigMaximilians-University of Munich, Germany. 1997. Veterinary and Comparative Orthopaedics and Traumatology.23(5), 297-305.

12. Olmstead, M. L., Hohn, R. B. \& Turner, T. M. (1983). A five-year study of 221 total hip replacements in the dog. Journal of the American Veterinary Medical Association. 183(2), 191-194.

13. Parker, R. B., Spencer, C. P., Bloomberg, M. S., Bitetto, W. \& Rodkey, W. G. (1984). Canine total hip arthroplasty: a radiographic correlation of clinical results in 20 cases. Journal of the American Animal Hospital Association. 20(1), 105-113.

14. Rawson, E. A., Aronsohn, M. G. \& Burk, R. L. (2005). Simultaneous Bilateral Femoral Head and Neck Ostectomy for the Treatment of Canine Hip Dysplasia. Journal of the American Animal Hospital Association. 41(3), 166-170. DOI: https://doi.org/10.5326/0410166

15. Vezzoni, L., Vezzoni, A. \& Boudrieau, R. J. (2015). Long-Term Outcome of Zürich Cementless Total Hip Arthroplasty in 439 Cases. Veterinary Surgery. 44(8), 921-929. DOI: https://doi.org/10.1111/vsu.12371 


\section{EVIIDEFeE

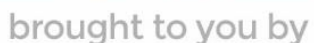 \\ RCVS KNOWLEDGE}

\section{Intellectual Property Rights}

Authors of Knowledge Summaries submitted to RCVS Knowledge for publication will retain copyright in their work, and will be required to grant RCVS Knowledge a non-exclusive license of the rights of copyright in the materials including but not limited to the right to publish, re-

publish, transmit, sell, distribute and otherwise use the materials in all languages and all media throughout the world, and to license or permit others to do so.

\section{Disclaimer}

Knowledge Summaries are a peer-reviewed article type which aims to answer a clinical question based on the best available current evidence. It does not override the responsibility

of the practitioner. Informed decisions should be made by considering such factors as individual clinical expertise and judgement along with patient's circumstances and owners' values. Knowledge Summaries are a resource to help inform and any opinions expressed within the Knowledge Summaries are the author's own and do not necessarily reflect the view of the RCVS Knowledge. Authors are responsible for the accuracy of the content. While the

Editor and Publisher believe that all content herein are in accord with current recommendations and practice at the time of publication, they accept no legal responsibility

for any errors or omissions, and make no warranty, express or implied, with respect to material contained within.

For further information please refer to our Terms of Use.

RCVS Knowledge is the independent charity associated with the Royal College of Veterinary Surgeons (RCVS). Our ambition is to become a global intermediary for evidence based veterinary knowledge by providing access to information

that is of immediate value to practicing veterinary professionals and directly contributes to evidence based clinical decision-making.

https://www.veterinaryevidence.org/

RCVS Knowledge is a registered Charity No. 230886.

Registered as a Company limited by guarantee in England and Wales No. 598443.

Registered Office: Belgravia House, 62-64 Horseferry Road, London SW1P 2AF

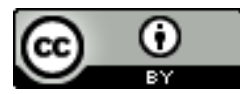

This work is licensed under a Creative Commons Attribution 4.0 International License 